\title{
Bogota Bag in Abdominal Compartment Syndrome at Kathmandu Model Hospital
}

\author{
Bijendra Dhoj Joshi, ${ }^{1}$ Udaya Koirala, ${ }^{1}$ Amit Mani Upadhyaya, ${ }^{1}$ Arbin Joshi, ${ }^{1}$ Saroj Dhital ${ }^{1}$ \\ ${ }^{1}$ Department of Surgery, Kathmandu Model Hospital, Exhibition Road, Kathmandu, Nepal.
}

\begin{abstract}
Background: The gold standard of quick and definitive treatment of Abdominal compartment syndrome (ACS) is surgical decompression by opening the abdomen and leaving it open until intra-abdominal pressure decreases. Temporary abdominal closure techniques are used to postpone definite closure until predisposing factors causing pathologic elevation of intra-abdominal pressure are resolved.This study aim to analyze feasibility of Bogota Bag placement as a way of temporary abdominal closure.

Methods: Cases admitted in the period of eight years that were diagnosed to have or at risk to develop ACS and managed with 'Bogota Bag', irrespective of primary diagnosis were reviewed retrospectively. Cause of ACS, reasons to place Bogota bag, its complications and final outcome in terms of mortality related or not related with Bogota Bag placement were assessed.

Results: Total of ten patients had placement of Bogota Bag in the period of eight years. Laparotomy for bowel perforation with peritonitis was the most common primary condition contributing to ACS. Bogota bag was placed in two cases after emergency decompression as a therapeutic measure whereas others were done as prophylactic measure. There were two mortalities (20\%) which were not directly related to abdominal compartment syndrome.

Conclusions: Abdomen closure with Bogota Bag for patients with ACS or likely to develop ACS is a feasible technique with minimal procedure related morbidities.

Keywords: Abdominal compartment syndrome; bogota bag; wound closure.
\end{abstract}

\section{INTRODUCTION}

Intra-abdominal hypertension (IAH) and Abdominal Compartment Syndrome (ACS) have been shown to occur with high prevalence in intensive care unit patients and causes significant mortality. ${ }^{1}$ Mortality is $26.1 \%$ for grade III abdominal compartment syndrome while it is $100 \%$ for grade IV. ${ }^{2}$ Mortality from ACS remains high (50\%-60\%) even when decompression of the abdomen is performed early, which highlights the importance of detection and treatment of IAH before end-organ damage occurs. ${ }^{2}$ Both 'conservative' and operative methods have been in use to cope with ACS with variable results. Use of 'Bogota Bag' is one of the cheapest and most efficient way of surgically dealing with ACS. The Columbian Surgical Resident Oswaldo Borraez ${ }^{3}$ was the first to use this technique with genitourinary irrigation bag. Bogota bag acts as a hermetic barrier that avoids evisceration and loss of fluids, and the abdominal contents can be visually inspected which is particularly useful in cases of ischemic bowel. Bag is inexpensive and easily available.

We aim to evaluate cases in which we used Bogota Bag as a temporary measure to close the abdomen and analyze the feasibility of this procedure.

\section{METHODS}

This was a retrospective study done in Kathmandu Model Hospital for the period of eight years (2008 January to 2015 December). Patients who had Bogota Bag placement during the study period were identified from electronic discharge datasheet and charts of these patients were retrieved from the record section. Those patients admitted for different surgical abdominal conditions who underwent decompressive laparotomy, who were diagnosed to have ACS (with the help of abdominal pressure measurement with the method as suggested by Kron et $\mathrm{al}^{4}$ - Fig 1) or were thought to be at risk of ACS during surgery and underwent Bogota bag 
placement were included in the study. Grade IV ACS is considered as indication for abdominal decompression and Bogota Bag placement. Transfusion bag was used as Bogota bag for the closure of abdomen (Fig 2 and 3). A short questionnaire was formulated to collect the data from the records. Patients with incomplete data in the chart were excluded. Ethical approval was obtained from Institutional Review Board of the hospital.

Variables like conditions causing ACS, reasons to place Bogota bag, morbidities and mortalities related or unrelated with placement of Bogota bag were analyzed.

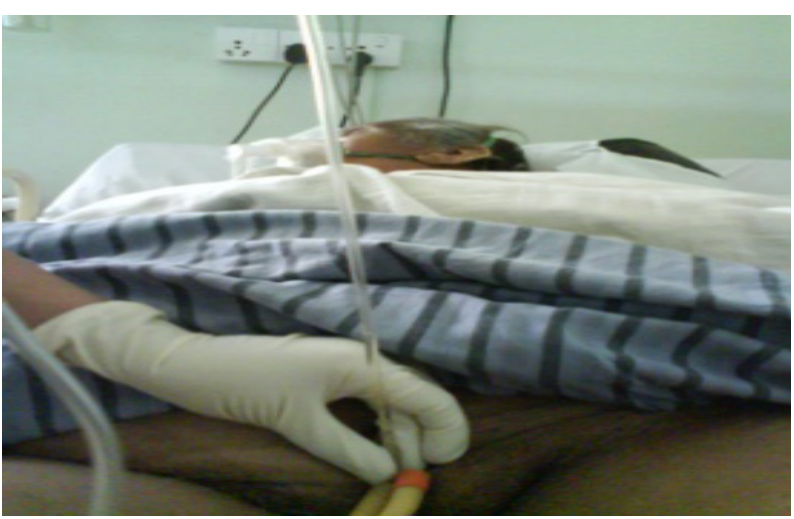

Figure 1.Transvesical measurement of intraabdominal pressure.

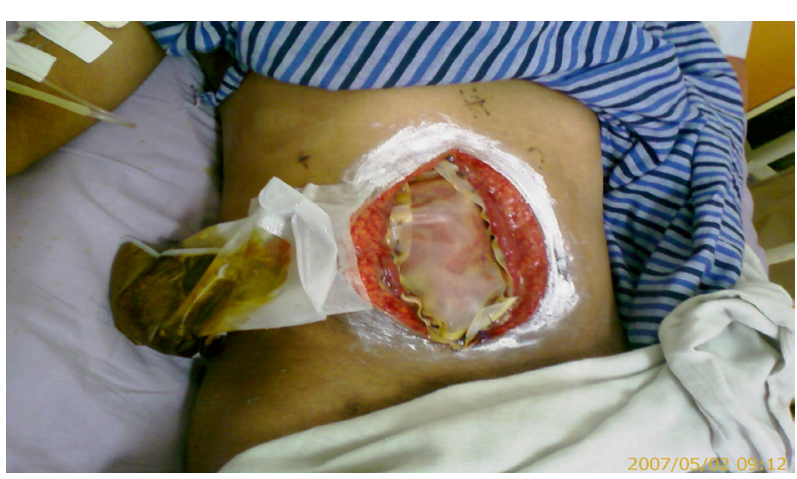

Figure 2. Bogota bag.

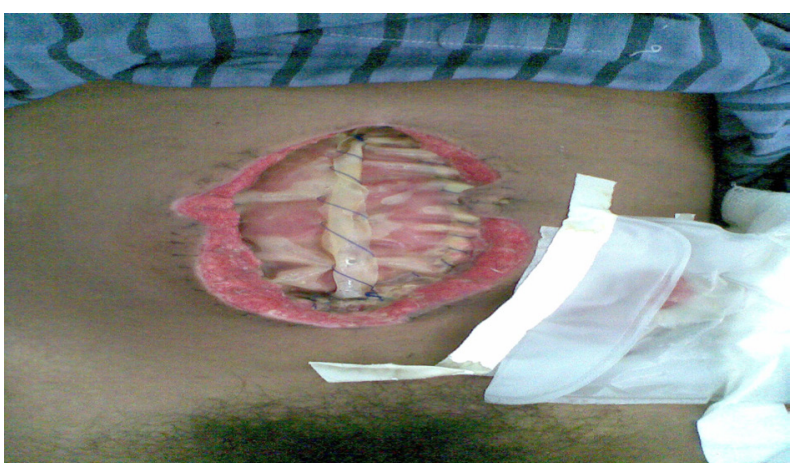

Figure 3. Bogota bag with ileostomy bag.

\section{RESULTS}

Total of 10 patients satisfied the inclusion criteria during the study period. Age of the patients ranged from 18 to 48 years $($ Mean $=35.2$ ). Male were $80 \%$ ( 8 out of 10$)$.

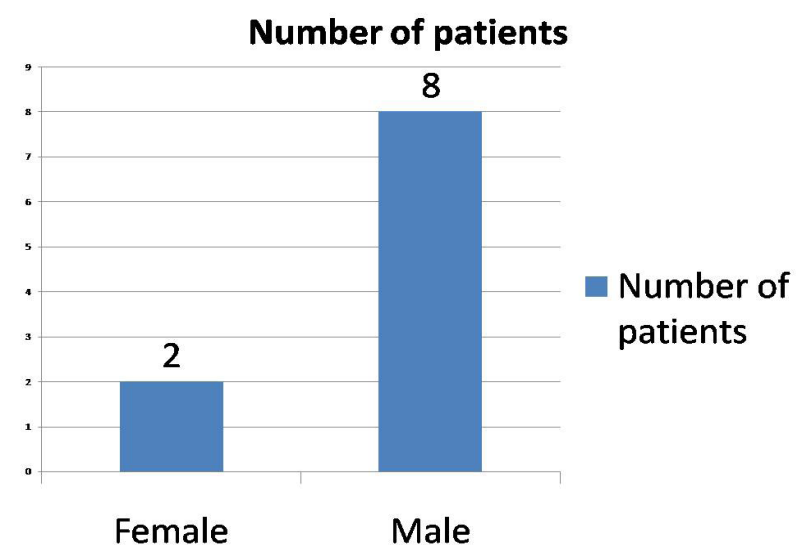

Figure 4. Sexwise distribution of the patients.

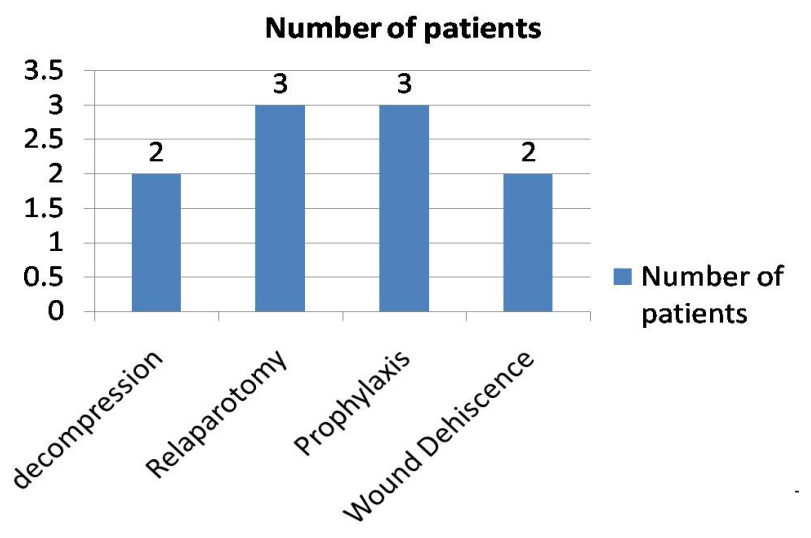

Figure 5. Reason to place Bogota Bag in different patients.

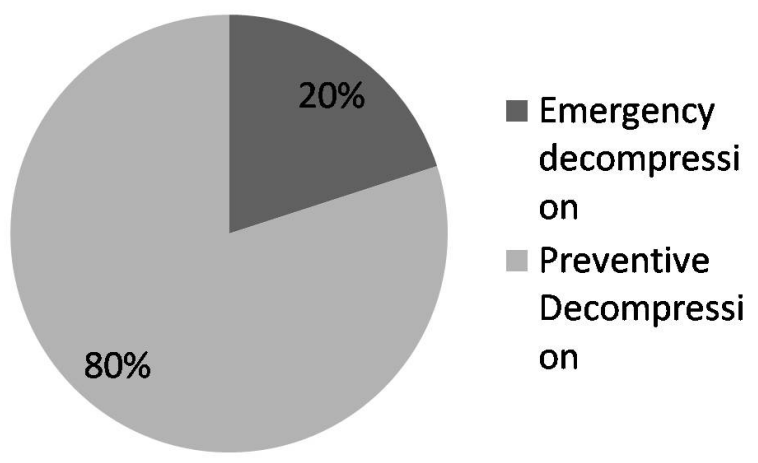

Figure 6. Emergency versus earlypreventive decompression. 


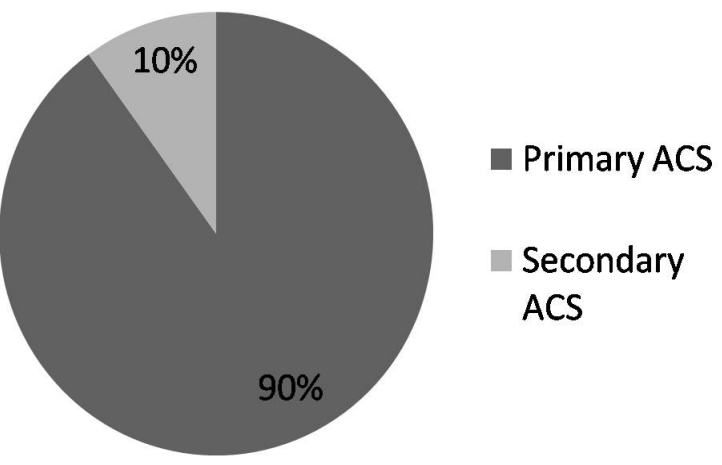

Figure 7. Primary ACS vs Secondary ACS.

Table 1. Causes for laparotomy in ten patients who

had Bogota Bag placed during eight year study period.

Disease

No. of patients

Small bowel volvulus

Enteric perforation

1

Secondary abdominal

compartment syndrome*

Traumatic small bowel

3

perforation

Caecal perforation

Strangulated femoral hernia

1

Acute necrotising pancreatitis*

1

*Patients who had grade IV ACS and underwent emergency decompression and Bogoto Bag placement

\section{DISCUSSION}

The ACS is a well established and studied phenomenon but may be the most ignored too. We undertook ten cases of Bogota Bag placement in last eight years which shows uncommon occurrence of this procedure. We had two patients who underwent emergency decompression. Remaining eight patients had preventive decompression whom we appreciated difficulty in closure and would have developed abdominal compartment syndrome if tension free measures were not used. Among emergency decompression, one patient was with secondary abdominal compartment syndrome who developed ascites and acute renal failure due to sepsis. This patient eventually survived, despite grade IV secondary abdominal compartment syndrome. Another patient who had undergone emergency decompression was the patient with acute necrotizing pancreatitis, also with grade IV abdominal compartment syndrome. Despite of the decompression this patient did not survive.
We used transvesical method to measure the intraabdominal pressure. This technique was first described by Kron and colleagues, who used a standard Foley catheter to measure the pressure in the bladder after instillation of sterile saline. ${ }^{3}$ Subsequent studies have confirmed the accuracy of this technique and shown it to be a reliable method of estimating the IAP. ${ }^{5}$ Prevention, diagnosis and early measurement of bladder pressure and a timely decompression are the keys to decrease the death rate in patients affected by this syndrome.

Abdominal decompression is a major undertaking and no consensus exists on after which grade of $\mathrm{IAH}$ decompressive laparotomy should be performed. It is a hazardous procedure, and full resuscitative facilities must be immediately available. Malignant arrhythmias have been reported on decompression, presumably secondary to the sudden efflux of products of anaerobic metabolism from the abdomen. ${ }^{4}$ After decompression, it is usual to adopt an 'open abdomen' technique and a number of methods have been described to achieve temporary abdominal closure. ${ }^{5}$ The management of the open abdomen is extremely challenging; early problems include infection and ongoing fluid losses, while fistulae and ventral hernias can lead to long-term complications. ${ }^{6}$ There is, therefore, an understandable reluctance amongst surgeons to perform this procedure. The majority of intensivists would recommend surgical decompression when the IAP exceeds $25 \mathrm{mmHg}$ and there is organ dysfunction $(64.4 \%, n=67)$. A further $27 \%(n=28)$ would use IAPs in excess of $20 \mathrm{~mm} \mathrm{Hg}$ with signs of organ dysfunction to recommend laparotomy. This is the approach recommended by the majority of authors, who suggest that patients with isolated IAH should be monitored closely for signs of physiological deterioration like hypotenstion, tachycardia, high respiratoy rate and poor urine output before proceeding toabdominal decompression. ${ }^{7,8,9}$ In a study by Walter et al. ${ }^{10}$ the mortality of the secondary ACS among the trauma patients was $38 \%$, comparable with other series, reporting mortalities of $67 \%$ to $71 \% .{ }^{11}$ In contrast, the mortality in their nontrauma patients was $100 \% .{ }^{10}$

Secondary ACS may be encountered by general surgeons in a variety of clinical scenarios; resuscitation from severe shock appears to be the critical factor. Early identification and abdominal decompression are essential. Our patient with secondary abdominal compartment syndrome [SACS] was referred late who was struggling to be alive in ICU care. The patient had sepsis secondary to endocarditis and had developed gross ascites resulting in grade IV abdominal compartment syndrome. The patient already had developed acute 
renal failure and decompressive laparotomy was a high risk but life saving procedure for her. The recognition of SACS, particularly in the nontrauma population, should heighten awareness of this physiologic entity in all intensive care unit patients. Nonsurgeon physicians and even many surgeons may either be unaware of current management strategies or may still be underestimating the incidence of intraabdominal hypertension in their critically ill patient. ${ }^{12,13}$ It is possible that the secondary ACS in patients with severe septic or cardiogenic shock represents irreversible systemic cellular decompensation, i.e., the first clinical sign of multiple organ failure. ${ }^{14}$ Benefits of laparotomy include facility of re-exploration, watching the abdominal contents, reduction of the risk of intra-abdominal hypertension, and fascial preservation for further closure of the abdominal wall. ${ }^{15}$

Towel clip closure, Bogota Bag, polyglycolic acid mesh, polyglactin 910 mesh, zipper and vacuum-assisted closure systems are available options in the temporary abdominal closure procedures. ${ }^{16}$ Temporary closure of the abdominal wall with Bogota bag in these patients is a simple and inexpensive method, and this transparent bag permits evaluation of the intraabdominal space. ${ }^{17}$ We used transfusion bag as Bogota bag which acts as a hermetic barrier that avoids evisceration and loss of fluids, the abdominal contents can be visually inspected which is particularly useful in many cases, is inexpensive and easily available. Transparency allowed observation, smooth surface prevented fistula and friendly moist environment was present for the viscera.

\section{CONCLUSIONS}

Bogota Bag application during abdomen closure for patients with diagnosis of ACS or likely to develop ACS is a feasible technique with minimal procedure related morbidities.

\section{REFERENCES}

1. Burch JM, Moore EE, Moore FA, Franciose R. The abdominal compartment syndrome. Surg Clin North Am. 1996 Aug 1;76(4):833-42. Link

2. Hunter JD, Damani Z. Intra-abdominal hypertension and the abdominal compartment syndrome. Anaesthesia. 2004;59(5):899-907. Link

3. Borraez OA. Abdomen abierto: la heridamás desafiante. Rev Colomb Cir. 2008;23(4):204-9.Link

4. Kron IL, Harman PK, Nolan SP. Measurement of intra- abdominal pressure as a criterion for abdominal reexploration. Ann Surg. 1984;199:28-30. Link

5. Iberti TJ, Lieber CE, Benjamin E. Determination of intra-abdominal pressure using a transurethral bladder catheter: clinical validation of the technique. Anesthesiology.1989;70(1):47-50.Link

6. Moore AF, Hargest R, Martin M, Delicata RJ. Intraabdominal hypertension and the abdominal compartment syndrome. Br J Surg. 2004;91(9):1102-10.Link

7. Mayberry JC. Prevention of the abdominal compartment syndrome.Lancet. 1999;354:1749-50

8. Schein M, Ivatury R. Intra-abdominal hypertension and the abdominal compartment syndrome. Br J Surg. 1998;85:1027-8.

9. Ivatury RR, Diebel L, Porter JM, Simon RJ. Intraabdominal hypertension and the abdominal compartment syndrome.Surg Clin North Am. 1997;4:783-99.Link

10. Biffl WL, Moore EE, Burch JM, Offner PJ, Franciose RJ, Johnson JL.Secondary abdominal compartment syndrome is a highly lethal event. Am J Surg. 2001;182(6):645-8. $\underline{\text { Link }}$

11. Kopelman T, Harris C, Miller R, Arrillaga A. Abdominal compartment syndrome in patients with isolated extraperitoneal injuries. JTrauma. 2000;49:744-9. $\underline{\text { Link }}$

12. Malbrain ML, Chiumello D, Pelosi P, Wilmer A, Brienza $\mathrm{N}$, Malcangi V, et al. Prevalence of intraabdominal hypertension in critically ill patients: a multicentre epidemiological study. Intensive Care Med. 2004;30:8229.Link

13. Kimball EJ, Rollins MD, Mone MC, Hansen HJ, Baraghoshi GK, Johnston C, et al. Survey of intensive care physicians on the recognition and management of intra-abdominal hypertension and abdominal compartment syndrome. Crit Care Med. 2006;34:2340-8.Link

14. Raeburn CD, Moore EE, Biffl WL, Johnson JL, Meldrum DR, Offner PJ, et al. The abdominal compartment syndrome is a morbid complication of post-injury damage control surgery. Am J Surg. 2001;182:542-46.Link

15. Kaplan M, Banwell P, Orgill DP, Ivatury RR, Demetriades $\mathrm{D}$, Moore FA, et al. Guidelines for the management of the septic open abdomen. Supplement to Wounds. 2005; 17:124.

16. Rutherford EJ, Skeete DA, Brasel KJ. Management of the patient with an open abdomen: techniques in temporary and definitive closure. Curr Probl Surg. 2004;41:821-76. 
$\underline{\text { Link }}$

17. Kirshtein B, Roy-Shapira A, Lantsberg L, Mizrahi S. Use of the "Bogota bag" for temporary abdominal closure in patients with secondary peritonitis. Am Surg. 2007;73:249-52.Link 